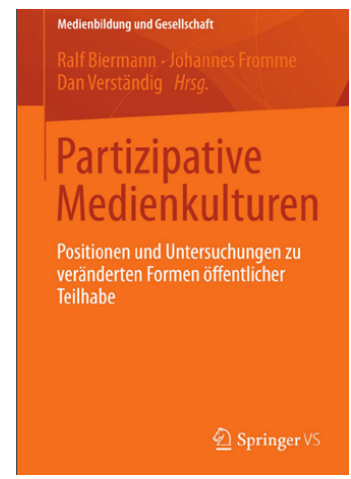

Ralf Biermann, Johannes Fromme, Dan Verständig (Hrsg.)

Partizipative Medienkulturen: Positionen und Untersuchungen zu veränderten Formen öffentlicher Teilhabe

Wiesbaden: Springer Fachmedien, 2014. 300 Seiten

ISBN: 3-658-01792-7

$€$ 39.99; CHF 50.-

\section{Partizipative Medienkulturen}

Das Einbringen des Begriffs der partizipativen Kultur in die wissenschaftliche Diskussion über Medien durch Henry Jenkins reicht in etwa so weit zurück wie die Etablierung der Medienpädagogik in Deutschland. Dennoch dauerte es bis ins Jahr 2012, ehe der Partizipationsbegriff erstmalig zum Themenschwerpunkt eines medienpädagogischen Theorieforums wurde. Der Sammelband Partizipative Medienkulturen - Positionen und Untersuchungen zu veränderten Formen öffentlicher Teilhabe, herausgegeben von Ralf Biermann, Johannes Fromme und Dan Verständig (alle Universität Magdeburg) präsentiert die Beiträge eben jenes Theorieforums und liefert gleichzeitig eine Zusammenschau des aktuellen Stands der Diskussion um partizipative Medienkulturen. Dabei wird in Rückgriff auf den Habermas'schen Partizipationsbegriff eine breite Palette an disziplinären Zugängen zusammengetragen, die sozial- und kulturwissenschaftliche, medien- und kommunikationswissenschaftliche sowie bildungs- und geisteswissenschaftliche Perspektiven umfasst.

Zunächst führen die Herausgeber mit einer eigenen, ausführlichen Einleitung in das Thema ein. Sie verdeutlichen, weshalb der Partizipationsbegriff auch viele Jahre nach Entstehung von Web 2.0 und Social Software noch ein zentrales Diskussionsthema ist. Die Herausgeber argumentieren, dass die Transformation von Beteiligungsmöglichkeiten im Zuge der fortschreitenden Mediatisierung ein gemeinsames Forschungsinteresse verschiedenster Disziplinen sein kann. Stellvertretend für die weiteren Autoren/-innen diskutieren sie Partizipation vor allem vor einem politischen Hintergrund, wobei sie mit Verweis auf die Cultural Studies auch weite Deutungen dessen, was als politisch zu verstehen ist, zulassen. Die Einleitung erscheint 
primär als Aufriss gedacht, um für die verschiedenartigen theoretischen und konzeptionellen Zugänge zu sensibilisieren, die in den Einzelbeiträgen folgen. Ungeachtet der gewünschten «Mannigfaltigkeit» gewährleistet die Einteilung in grundlegende und bereichsspezifische Beiträge einen roten Faden, der die Leserinnen und Leser durch das Buch führt.

Grundlegende Beiträge zu medialer und politischer Partizipation

Heinz Moser eröffnet die Einzelbeiträge mit einem Text zur Veränderung der politischen Teilnahme und Partizipation im Zeitalter digitaler Netze. Dabei zeigt er anhand der Erkenntnisse verschiedener Studien sehr anschaulich Wege auf, wie medienpädagogische Arbeit zu mehr politischer Beteiligung führen kann. Im amerikanischen Konzept der «Digital Citizenship» sieht er einen vielversprechenden Anknüpfungspunkt, sofern dieser nicht auf seine bewahrpädagogischen Akzentuierungen reduziert und die «Spielregeln» der Netzgemeinschaft stattdessen im Dialog der Lehrenden mit den Lernenden erkundet werden. Mit Wengers "Community of Practice»-Konzept identifiziert Moser zudem einen griffigen konzeptionellen Anker für die schrittweise Beteiligung und das «Hineinwachsen» in partizipative (Netz-)Kulturen, wobei der Lebensstil und die Interessen der jüngeren Generation aufgegriffen und vor dem Hintergrund ihrer politischen Bedeutung reflektiert werden.

Dass mediatisierte Partizipation nicht immer positiv zu sehen ist, sondern durchaus auch ihre Schattenseiten hat, beleuchtet der Beitrag von Jeffrey Wimmer am Fallbeispiel der Kampagne KONY 2012. Diese sollte zur Ergreifung des mutmasslichen Kriegsverbrechers Joseph Kony in Uganda führen und stand wegen falscher und vereinfachender Darstellung in der Kritik. Dennoch erlangte sie eine hohe Popularität und gilt als Paradebeispiel für eine reichweitenstarke Social-Media-Kampagne. Wimmer diskutiert diese Dynamiken und Problematiken neu entstehender Beteiligungsformen im Kontext der Mediatisierungsforschung. Er argumentiert, dass sich die Bedeutung von politischer Partizipation vor dem Hintergrund einer veränderten Kommunikationspraxis wandelt und neue Ausprägungen auftreten.

Im Beitrag von Christian Swertz wird dazu angeregt, sich kritisch mit der Verwendung des Begriffs «Partizipation» in Bildung und Politik auseinanderzusetzen. Er betont, dass der Partizipationsbegriff dem Begriff der «Emanzipation», der zuvor im medienpädagogischen Zielverständnis vorherrschte, nicht unbedingt nahe steht und weit weniger normativ geprägt ist. Während Emanzipation die Herauslösung aus tradierten Machtstrukturen betont, erscheint Partizipation vor dem Hintergrund der Einpassung in Strukturen als 
konservativer und - Zitat des Autors - «nahezu antidemokratisch» (S. 83). Hier könnte man durchaus gegenteilig argumentieren, wenn man annimmt, dass Partizipation im Diskurs mit Andersdenkenden funktioniert, wohingegen Emanzipation auch narzisstische Züge annehmen kann, was bei selbstbestimmt gelebter Partizipation wiederum schwer vorstellbar ist. In Bezug auf die sozialen Medien konstatiert der Autor, dass vor allem verschiedene Alternativen der Nutzung gelehrt werden sollten und dass ein «blosses Mitmachen» auf Facebook \& Co zu kurz greife. Auch wenn Selbstbestimmung in den Netzwerken (und nicht nur dort) ohne den gesamtgesellschaftlichen Horizont einer objektiven Realität passiert, so kann die Teilnahme im Sinne einer Veranschaulichung und Konstruktion von Medienutopien durchaus als medienpädagogische Zielsetzung nützlich sein, wobei Swertz in Bezug auf die Praxis insbesondere für eine eigene Gestaltung von Software und den Verzicht auf kommerzielle Dienste (Facebook, Google etc.) plädiert.

Jakob Dörre und Gerhard Chr. Bukow ziehen in ihrem Beitrag das Fazit, dass die Änderung von Beteiligungsmöglichkeiten durch das Netz zwar nicht - wie oft behauptet - von grundlegender Art, aber dennoch wissenschaftlich interessant sei. Sie stellen die These auf, dass partizipative Demokratieformen schon immer ihre natürlichen Grenzen hatten, welche durch die technologische Entwicklung unberührt bleiben. Wichtiger erscheint innen die Verantwortung der Technologieentwickler/innen, die selbst Forscher/innen bezüglich der Relevanz von Informationen und der bei Rezipienten/-innen ablaufenden Wahrnehmungs- und Meinungsbildungsprozesse sein können (und sollten?). Dieses Forschungsverständnis sehen die Autoren nicht nur vor dem Hintergrund von Effizienzgesichtspunkten bzw. um immer relevantere Informationen zu präsentieren. Vielmehr fordern sie, dass über die Filter- und Aggregationsmöglichkeiten und ihre normativen Implikationen reflektiert wird und dass diese in Bildungsprozesse eingehen - eine sehr wichtige Forderung, wenn man bedenkt, dass marktorientierte Technologieentwickler/innen genau hieran in der Regel wenig Interesse zeigen, da sie i.d.R. die Hoheit über ihre Algorithmen behalten möchten.

Erkenntnisse aus einem konkreten Forschungsprojekt zu Partizipation werden in dem Beitrag von Corinne Büching, Julia Walter-Herrmann und Heidi Schelhowe präsentiert. In einer Studie setzten sie sich mit der Erweiterung der von Henry Jenkins formulierten New Media Literacy um technologische Aspekte auseinander und stellen vier $\mathrm{m}$. E. hochinteressante Lernszenarien bzw. Workshops vor, die jeweils unterschiedliche Zugänge zum Lernen in einer partizipativen Kultur repräsentieren. Als Leser wünscht man sich je- 
doch an manchen Stellen mehr Abbildungen, um den komplexen Aufbau der Workshop-Settings zu durchdringen. Die Autorinnen schliessen ihren Beitrag mit der nachvollziehbaren, wenngleich nicht völlig neuen Feststellung, dass digitale Medien durch ihre Programmiertheit und ihre zumindest implizit enthaltene Aufforderung zum Mitgestalten mehr als nur «Tools» seien, da sie selbst Handlungsmacht besitzen, um partizipative Prozesse zu steuern. Ähnlich wie Christian Swertz schlussfolgern sie für Bildung, dass die im Hintergrund wirkenden technologischen Vorgänge und Algorithmen von Lernenden durchdrungen werden sollten. Wichtig erscheint ihre Feststellung, dass dies bei komplexen Systemen nicht zur Gänze möglich ist, sondern eher einem wechselseitigem Eintauchen und Verstehen gleicht.

Auch die historische Perspektive auf Partizipation kommt im Buch nicht zu kurz, wenn Thorsten Lorenz in seinem Beitrag die These aufstellt, dass insbesondere die Massenmedien seit jeher ihre (nicht unkritischen) Mechanismen haben, um unbekannte Akteure und Gruppen an der öffentlichen Meinung partizipieren zu lassen: Inwiefern das Verhältnis von Authentizität und Inszeniertheit einer solchen Partizipation in den sozialen Medien anders und vielleicht dialogorientierter verfasst ist, bleibt ein offener Diskussionspunkt.

\section{Bereichsspezifische Zugänge zu partizipativen Medienkulturen}

Im zweiten Teil des Sammelbands findet sich ein bunter Strauss an Beiträgen aus verschiedenen Kontexten, in denen partizipative Medienkulturen beobachtet werden können. Im ersten Beitrag von Karin Bruns steht das Verhältnis von Medien und Politik im Mittelpunkt, wobei speziell das so genannte "Leaking» und die Verbreitung von Gerüchten und Geheimnissen via Social Media erörtert werden. Es folgt ein bildungsbezogener Beitrag von Markus Deimann über die Zusammenhänge zwischen offenen Bildungsressourcen (OER) und Partizipation, bevor im Text von Benjamin Beil Bezüge zwischen der Computer-Spiele-Industrie und partizipativen Prozessen am Phänomen des «Moddings», also der Veränderung von Spielinhalten durch die Spieler/innen, hergestellt werden. Marten und Kristina Jonas ergänzen den Aspekt des Spielens um eine handlungsorientierte medienpädagogische Fragestellung, wenn sie in ihrem Beitrag erörtern, wie Computerspiele und deren Produktion Eingang in die aktive Medienarbeit finden können. Einen breiten Blick auf didaktische Fragestellungen nimmt schliesslich Kerstin Mayrberger ein, die diskutiert, ob das Forschungs- und Handlungsfeld der mediendidaktischen Disziplin um die spezifische Form einer «partizipativen Mediendidaktik» erweitert werden sollte. Ihr Beitrag liefert einen konzeptionellen 


\section{Rezensionen}

Grundrahmen, der aufzeigt, wie eine solche Mediendidaktik zukünftig aussehen kann. Das Buch schliesst mit einem Text von Tobias Hölterhof und Mandy Schiefner-Rohs, die insbesondere Peer-Education als Möglichkeit diskutieren, in formalen Bildungskontexten Raum für Authentizität und Selbstbestimmung zu wahren.

Durch die Beiträge im zweiten Teil zieht sich trotz ihrer grossen thematischen Breite ein roter Faden, der von allgemein gesellschaftlichen hin zu bildungsbezogenen Fragestellungen führt und zu guter Letzt die Didaktik und Gestaltung von (partizipativen) Bildungskontexten fokussiert.

\section{Gesamtbetrachtung}

Mit dem vorgelegten Sammelband gelingt es den Herausgebern, theoretische Reflexionen mit praktischen Forschungsergebnissen zu verknüpfen, und er bietet auf diese Weise einen verständlichen und gut lesbaren Zugang in die Partizipationsthematik. Die Strukturierung in allgemeine und bereichsspezifische Beiträge erscheint logisch und hilft zur Einordnung, auch wenn die Kriterien für die Auswahl und den Einbezug von Anwendungskontexten in den Diskurs für den zweiten Teil des Buches nicht explizit gemacht werden. Vor allem kann die Tatsache überzeugen, dass tatsächlich kontrasträre Positionen zu der grundlegenden Bedeutung technologischer Veränderungen im Hinblick auf Partizipationskulturen referiert werden. Es hätte sich unter Umständen noch angeboten, die Diskussionen des Forums stärker in dialogischer Form darzubieten und beispielsweise Interviews, Streitgespräche oder Gegenüberstellungen in das Buch einfliessen zu lassen. So bleibt es dem Leser und der Leserin überlassen, die dargebotenen Positionen, die in ihrer Vielfalt zentrale Fragen bzgl. partizipativer Medienkulturen aufwerfen und an geeigneter Stelle vertiefen, in Bezug zu setzen.

Philip Meyer 